\title{
A Pore Residue of the KCNQ3 Potassium M-Channel Subunit Controls Surface Expression
}

\author{
Juan Camilo Gómez-Posada, ${ }^{1 \star}$ Ainhoa Etxeberría, ${ }^{1 \star}$ Meritxell Roura-Ferrer, ${ }^{1}$ Pilar Areso, ${ }^{2}$ Marianela Masin, ${ }^{3}$ \\ Ruth D. Murrell-Lagnado, ${ }^{3}$ and Alvaro Villarroel ${ }^{1}$ \\ ${ }^{1}$ Unidad de Biofísica, Centro Mixto Consejo Superior de Investigaciones Científicas-Universidad del País Vasco/Euskal Herriko Unibertsitatea (UPV/EHU), \\ and ${ }^{2}$ Departamento de Farmacología, UPV/EHU, 48940 Leioa, Spain, and ${ }^{3}$ Department of Pharmacology, University of Cambridge, Cambridge CB2 1PD, \\ United Kingdom
}

KCNQ2 (Kv7.2) and KCNQ3 (Kv7.3) are the principal subunits underlying the potassium M-current, which exerts a strong control on neuronal excitability. KCNQ3 subunits coassemble with KCNQ2 to form functional heteromeric channels that are specifically transported to the axonal initial segment and nodes of Ranvier. In contrast, there is no evidence for functional homomeric KCNQ3 channels in neurons, and it appears that these are inefficiently trafficked to the plasma membrane. Among eukaryotic potassium channels, the $\mathrm{KCNQ} 3$ subunit is unusual because it has an alanine in place of a threonine at the pore inner vestibule, three residues upstream of the GYG signature sequence of the selectivity filter. This residue is critical for the potentiation of the current after heteromerization, but the mechanism is unknown. We report that the presence of this uncommon residue at position 315 has a strong impact on the stability of the homotetramers and on channel trafficking. Wild-type KCNQ3 expressed alone is retained within the endoplasmic reticulum, and this mechanism is overcome by the substitution of threonine for Ala315. KCNQ3 subunits require assembly with KCNQ2 to exit this compartment, whereas KCNQ3-A315T is no longer dependent on KCNQ2 to form channels that are efficiently trafficked to the plasma membrane. The presence of this alanine, therefore, plays an important role in regulating the subunit composition of functional M-channels expressed at the surface of neurons.

\section{Introduction}

Voltage-gated KCNQ (Kv7) potassium channels are of critical importance in excitable tissue (Jentsch, 2000). In the nervous system, KCNQ2 and KCNQ3 subunits are the main component of the M-current (Wang et al., 1998; Shah et al., 2002), which is a voltage-dependent, non-inactivating potassium current that plays a central role in integrating activity of both peripheral and central neurons. From an evolutionary perspective, KCNQ2 and KCNQ3 were the last KCNQ subunits to emerge, coincident with the apparition of myelin (Hill et al., 2008). The KCNQ2/3 subunits acquired an ankyrin G-binding motif, that allows them to concentrate at the nodes of Ranvier and the axonal initial segment (AIS) (Pan et al., 2006; Rasmussen et al., 2007).

Spontaneous mutations in these subunits, some producing a mere 25\% reduction in function, cause human neonatal epilepsy (Schroeder et al., 1998; Maljevic et al., 2008). The relative contri-

\footnotetext{
Received Feb. 4, 2010; revised May 7, 2010; accepted May 26, 2010.

This work was supported by grants from the VII European framework program managed by the Fondo de Investigaciones Sanitarias (PI071316) and from the Spanish Ministry of Education (BFU2009-07581 and SAF20061450) and the Spanish lon Channel Initiative Consolider project (CSD2008-00005). J.C.G.-P. held an I3P Consejo Superior de Investigaciones Científicas fellowship cofinanced by the Fondo Social Europeo.

*J.C.G.-P. and A.E. contributed equally to this work.

Correspondence should be addressed to Alvaro Villarroel, Unidad de Biofísica, Centro Mixto CSIC-UPV/EHU, Universidad del País Vasco, Barrio Sarriena s/n, 48940 Leioa, Spain. E-mail: gbxvimua@ehu.es.

A. Etxeberría's present address: Department of Neurology, Center for Neuroscience Research, Children's National Medical Center, Washington, DC 20010.

DOI:10.1523/JNEUROSCI.0851-10.2010

Copyright $\odot 2010$ the authors $\quad 0270-6474 / 10 / 309316-08 \$ 15.00 / 0$
}

bution of any ion channel to neuronal excitability is primarily determined by their functional characteristics, abundance, and distribution at the plasma membrane (Misonou and Trimmer, 2004). KCNQ2 and KCNQ3 subunits combine in equal stoichiometric proportions in neurons (Hadley et al., 2003) by a mechanism that is not fully understood. The understanding of how this specific combination is favored is important since such combination affect the properties of the channels. Expression of KCNQ2 and KCNQ3 yields only small currents, whereas their coexpression yields heteromeric currents 10-fold larger (Wang et al., 1998). KCNQ3 is a major determinant of M-channels location to the AIS (Rasmussen et al., 2007) and displays a predominant intracellular distribution, whereas its combination with KCNQ2 leads to a large increase in axonal surface expression (Chung et al., 2006).

KCNQ3 is unusual because it has an alanine in the inner vestibule, three residues upstream of the signature GYG (see Fig. $1 A)$. It is present in all KCNQ3 tetrapod orthologs, but not in fishes. This amino acid plays a critical role in inhibiting the function of KCNQ3 homomeric channels (Etxeberria et al., 2004), but the mechanism is unknown. It is generally thought that KCNQ2/3 surface expression is governed by the intracellular C-terminal region, and that the pore region does not have any significant role in trafficking (Maljevic et al., 2003; Schwake et al., 2003, 2006; Etxeberria et al., 2004; Nakajo and Kubo, 2008; Zaika et al., 2008). This presumption and the observation that KCNQ3A315T is functional have led to the suggestion that KCNQ3 may 
A

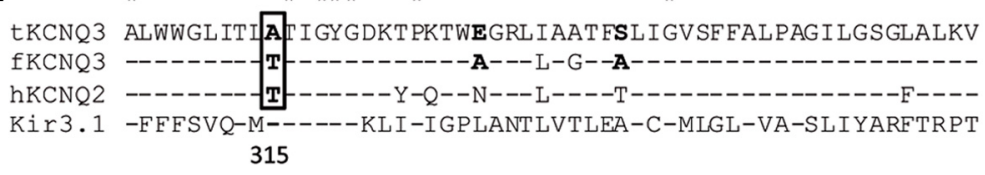

B

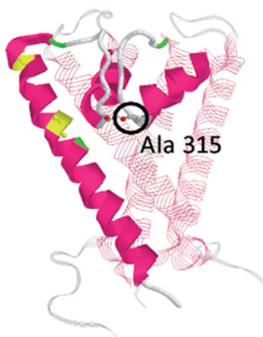

D

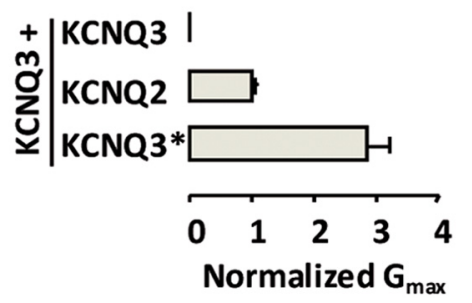

C

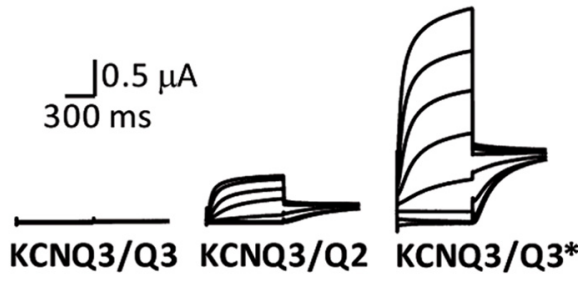

$\mathbf{E}$

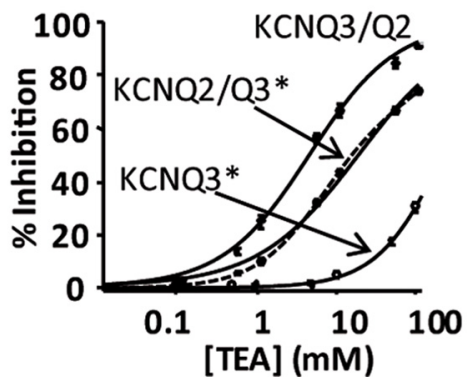

Figure 1. The presence of Ala315 makes the function of KCNQ3 subunits dependent on the association with KCNQ2 subunits. A, Sequence alignment of the pore region investigated in this study of tetrapod (tKCNQ3) and fish (fKCNQ3), compared to human KCNQ2 (hKCNQ2) and Kir3.1. Searching the Ensembl database (http://aug2007.archive.ensembl.org/index.html), we found alanine at the equivalent position to be present in all KCNQ3 tetrapod orthologs, but not in fishes (see supplemental Fig. S1, available at www.jneurosci.org as supplemental material). In contrast, an alanine is present in the inner vestibule of bacterial, fish, and tetrapod Kir3.1 subunits. The stars indicate residues conserved. The numeration is based on human KCNQ3. B. Structure of a Kir3.1 chimera (1XL4) (Nishida et al., 2007). Only two subunits are displayed for clarity. The selectivity filter of both subunits and the TM2 (equivalent to S6 of KCNQ3) are rendered as a diagram using Rasmol. C, Representative collection of currents recorded in oocytes at $-20 \mathrm{mV}$ evoked after $800 \mathrm{~ms}$ voltage steps from $-120 \mathrm{mV}$ to $+50 \mathrm{mV}$. The holding potential was $-50 \mathrm{mV}$. D, Averaged normalized maximal conductance obtained after fitting a Boltzmann distribution to $g-V$ relationships from tail currents measured at $-20 \mathrm{mV}(n>8)$. The difference between KCNQ2 and KCNQ3* is statistically significant ( $p<0.002$, unpaired Student's $t$ test). $E$, Dose-response curves for TEA blockade of the currents recorded from oocytes expressing the combination of subunits indicated (1:1 ratio). The solid lines are the result of fitting a one-population Hill equation to the data with an $\mathrm{IC}_{50}$ value of $\sim 4.1 \mathrm{~mm}$ for KCNQ2/3 and $\sim 223.5 \mathrm{~mm}$ for KCNQ3*. The discontinuous line is the result of fitting a two-population Hill equation to the data from KCNQ2/3*-expressing 0ocytes. The fit of a two-population equation was significantly better than the fit of a one-population Hill equation ( $p<0.001$ ). A population representing $69 \%$ of the channels had an $\mathrm{IC}_{50}$ value of $6.1 \mathrm{~mm}$, and the remaining $31 \%$ of the channels had an $\mathrm{IC}_{50}$ value of $239.2 \mathrm{~mm}(n>8)$.

be present at the plasma membrane in an electrically silent conformation (Etxeberria et al., 2004; Zaika et al., 2008).

Here we have investigated the impact of this residue on channel assembly and trafficking. Against expectations, we find that Ala315 is a major trafficking determinant. KCNQ3 subunits are retained at the endoplasmic reticulum (ER), whereas the KCNQ3A315T mutation increases the stability of the homotetramers and trafficking to the cell surface. Our results indicate that KCNQ3 channels are intrinsically unstable, a property that is critical for their dependence on heteromultimerization.

\section{Materials and Methods}

Molecular biology. Human KCNQ2 (Y15065) and KCNQ3 (NM004519) cDNAs were used. For immunoprecipitation and imaging experiments, we used the KCNQ3 subunit tagged at the N-terminal with mYFP (citrine) and cloned into pCDNA3.1 (Invitrogen). The ER marker pDsRed2-ER was obtained from Clontech. For Western blotting, we used, unless stated otherwise, the KCNQ3 subunit tagged at the
$\mathrm{N}$-terminal with a tandem repeat of $2 \mathrm{HA}$ epitopes (YPYDVPDYA) and the KCNQ2 subunit tagged at the N-terminal with a tandem repeat of 5 myc epitopes (MEQKLISEEDLN). Those constructs were subcloned into pCAGGS. The electrophysiological properties recorded in Xenopus oocytes of tagged KCNQ3 and KCNQ2 constructs were indistinguishable from the nontagged equivalents.

Antibodies. The antibodies used here included the following: a rat monoclonal anti-HA (3F10; Roche Diagnostics), a mouse polyclonal antimyc (9E10; Sigma-Aldrich), and a mouse monoclonal anti-GFP (clones 7.1 and 13.1; Roche). The peroxidase-coupled secondary antibodies used were an anti-mouse IgG (Bio-Rad Laboratories) and an anti-rat IgG (Jackson ImmunoResearch Laboratories).

Electrophysiological measurements. Standard two-electrode voltage-clamp procedures were used to record currents from Xenopus oocytes (Etxeberria et al., 2004). To monitor the assembly of KCNQ3 with KCNQ2 and KCNQ3*, sensitivity to blockage by tetraethylammonium (TEA) was measured (Etxeberria et al., 2004). Currents were elicited by an $800 \mathrm{~ms}$ depolarizing step from -120 to $+50 \mathrm{mV}$ every $20 \mathrm{~s}$. Data were normalized in Excel (Microsoft) and plotted in SigmaPlot (SPSS).

TEA concentration-inhibition curves were initially fitted with the Hill equation:

$$
y / y_{\max }=1\left[1+\left(x / x_{0}\right)^{p}\right],
$$

where $y$ is the relaxation amplitude; $y_{\max }$ is the full inhibition; $x$ is the concentration of the blocker; $x_{0}$ is the $\mathrm{IC}_{50}$ (the concentration at which $y / y_{\max }=0.5$ ); and $p$ is the power (equivalent to the Hill slope, $n_{\mathrm{H}}$ ). For the twocomponent fit to TEA inhibition, a modified equation was applied in SigmaPlot. This had the following form:

$$
\begin{aligned}
y / y_{\max }=q\left[1+\left(x / x_{0}\right)^{p 0}\right]+ & (1-q) /[1 \\
& \left.+\left(x / x_{1}\right)^{p 1}\right],
\end{aligned}
$$

where $x_{0}$ and $x_{1}$ are the $\mathrm{IC}_{50} \mathrm{~s}$ for two channel populations with proportional contributions of $q$ and $(1-q)$, respectively.

The factor $p$ was not included because we assumed the presence of separate channel populations each with a Hill slope of unity. In all cases, $y_{\max }$ was taken to be $100 \%$, even if full inhibition was not obtained at the highest TEA concentration used. Prism 5.01(GraphPad Software) was used for statistical comparison of the fit to alternative equations.

Surface expression. A KCNQ3 subunit tagged with an HA epitope in the extracellular loop connecting the S1 and S2 transmembrane domains was used in chemiluminescent assays of individual oocytes (Schwake et al., 2000; Etxeberria et al., 2004). Background signal obtained from uninjected oocytes was subtracted (background represented $<10 \%$ of the maximal signal).

Cell culture and transfection. DNA from each construct was transfected overnight by the calcium phosphate method into HEK293T cells that were maintained in DMEM medium (Sigma) supplemented with nonessential amino acids (Sigma) and 10\% FBS (Invitrogen) for 24-36 h.

Immunoprecipitation and immunoblotting. HEK293T cells were solubilized for $30 \mathrm{~min}$ at $4^{\circ} \mathrm{C} 24 \mathrm{~h}$ after transfection in IP buffer containing Tris- $\mathrm{HCl} 50$ mм, NaCl 150 mm, 1\% Triton X-100, EDTA 2 mм, EGTA 5 $\mathrm{mm}$, and protease inhibitors $(1 \times$ Complete; Roche). The nuclei were 
pelleted at $500 \times g$ for $3 \mathrm{~min}$, followed by centrifugation at $15,000 \times g$ for $10 \mathrm{~min}$ to remove the insoluble material. Lysates were precleared with 40 $\mu \mathrm{l}$ of equilibrated Protein A Sepharose beads (Sigma P3391) for $1 \mathrm{~h}$ at $4^{\circ} \mathrm{C}$. Anti-GFP antibodies were immobilized with $40 \mu \mathrm{l}$ of equilibrated Protein A beads overnight at $4^{\circ} \mathrm{C}$ and washed twice with IP buffer. Precleared lysates were incubated with Protein A-anti-GFP for $4 \mathrm{~h}$ at $4^{\circ} \mathrm{C}$ and, after four washes with IP buffer, immunoprecipitated proteins were recovered after heating at $90^{\circ} \mathrm{C}$ for $5 \mathrm{~min}$ in SDS sample buffer. For quantitative immunoblot analysis, total protein was separated by SDSPAGE, probed with anti- $\alpha$ tubulin (DM1A; Sigma), and quantified using ImageJ software. Sample load was then adjusted based on the tubulin levels detected.

Blue native-PAGE. Cells were washed with ice-cold PBS containing protease inhibitors, harvested, and lysed in hypotonic buffer $[10 \mathrm{mM}$ Tris-HCl, pH 8.0, 2 mm EDTA, 1 mm PMSF, and protease inhibitor cocktail (Roche)]. Cells were further disrupted by repeated passes through 23 and 27 gauge needles. The homogenate was centrifuged at $560 \times g$ for $10 \mathrm{~min}$ at $4^{\circ} \mathrm{C}$ to remove nuclei and then spun at $50,000 \times g$ for $35 \mathrm{~min}$ at $4^{\circ} \mathrm{C}$. The pellet was resuspended in blue native (BN) sample buffer (Invitrogen) and solubilized in 1\% digitonin for $1 \mathrm{~h}$ on ice. For partial denaturation, samples were subsequently incubated with $0.05 \%$ SDS at $37^{\circ} \mathrm{C}$ for $1 \mathrm{~h}$. Solubilized membrane fractions were supplemented with $0.25 \%$ G250 additive buffer (Invitrogen) and centrifuged at $15,000 \times g$ for $10 \mathrm{~min}$ at $4^{\circ} \mathrm{C}$. Samples were run on $4-16 \%$ ready-made Bis-Tris gels (Invitrogen), transferred onto PVDF membranes, and immunoblotted with appropriate antibodies. Triton X-100 extracts were lysed in $50 \mathrm{~mm}$ BisTris, pH 7.0, 1\% Triton X-100, $600 \mathrm{~mm}$ aminocaproic acid, and $1 \mathrm{~mm}$ EDTA supplemented with inhibitor cocktail. The loading buffer $(5 \times)$ was $250 \mathrm{~mm}$ Tris- $\mathrm{HCl}, \mathrm{pH}$ 6.8, Coomassie blue G 0.5\%, glycerol 50\%, SDS $1 \%$ ([SDS] final $0.2 \%$ ). Running buffer was $25 \mathrm{~mm}$ Tris Base, $200 \mathrm{~mm}$ glycine, 0.01\% SDS, and Coomassie blue G 0.002\%, $\mathrm{pH} 7.0$.

Hydrodynamics methods. HEK293T cells from a flask T-75 were lysed in $250 \mu$ l of digitonin lysis buffer [ $150 \mathrm{~mm} \mathrm{NaCl}, 20$ mм HEPES, pH 7.4, $2 \mathrm{~mm}$ EDTA, 5\% glycerol, $25 \mathrm{~mm}$ iodoacetamide, $1 \times$ protease inhibitor cocktail (Roche), $100 \mu \mathrm{g} / \mathrm{ml} \mathrm{PMSF}$, and $1 \%$ digitonin] during $1 \mathrm{~h}$ at $4^{\circ} \mathrm{C}$, then were cleared at $16,000 \times g$ for $30 \mathrm{~min}$ at $4^{\circ} \mathrm{C}$. Soluble material was layered onto $10-50 \%$ glycerol gradients (glycerol w/v, $100 \mathrm{~mm} \mathrm{NaCl}, 20$ mM HEPES, pH 7.4, and 2 mM EDTA). Gradients were made with a Gradient Master 107 (Biocomp Instruments) and centrifuged in a TST 41.14 Centrikon rotor (an equivalent of SW40 rotor of Beckman Coulter) at $200,000 \times g$ for $18 \mathrm{~h}$ at $4^{\circ} \mathrm{C}$, with brakes fully applied (maximum acceleration, maximum deceleration). Sixteen fractions of $750 \mu \mathrm{l}$ were collected manually from the top.

Image acquisition and analysis. Colocalization experiments were performed using a Nikon TE2000-U microscope equipped for epifluorescence and with a Nikon D-eclipse C1 Si confocal spectral detector, using a $60 \times, 1.45$ numerical aperture, oil-immersion objective as described previously (Etxeberria et al., 2008). The fluorescent proteins were excited using a $488 \mathrm{~nm}$ laser line, and 32 images were acquired simultaneously at different emission wavelengths between 450 and $610 \mathrm{~nm}$ at $5 \mathrm{~nm}$ intervals. For each cell, 10 optical sections (in $0.6 \mu \mathrm{m} Z$-steps) were acquired using a $30 \mu \mathrm{m}$ diameter pinhole, and cells with similar levels of expression (as judged by brightness) were selected for analysis. The signal from each fluorophore was isolated by performing spectral unmixing using the EZ C1 3.00 software (Nikon) (Etxeberria et al., 2008). The reference spectra were acquired from cells expressing YFP-KCNQ3 and pDsRed2-ER alone. Images were analyzed for colocalization using ImageJ software (version WCIF, National Institutes of Health), using the plug-in to calculate the Manders coefficient (Manders et al., 1992). Images were converted to $8 \mathrm{bit}$, and the perimeter of the cell was drawn manually for analysis. The correct identification of colocalized pixels pairs is essential to the accurate index evaluation, and it is accomplished by defining a threshold value for each detection channel. Specific pixels will contribute to the colocalized signal only if both channel intensities are above their respective threshold values. For a particular channel, the ratio of the colocalized pixel intensities to the total pixel intensities, define the interacting fraction for that species. Before Manders analysis, the background was automatically evaluated and subtracted (Costes et al., 2004). Similar indexes for each image of the stack from selected cells were obtained. The third image from the stack, counting from the bottom of the cell, was analyzed for colocalization.

Total internal reflection fluorescence (TIRF) images were collected at room temperature using an inverted TE2000-U microscope with through-the-lens (prismless) TIRF imaging, equipped with a plan Apo TIRF $60 \times$ oil-immersion high-resolution (1.49 numerical aperture) TIRF objective. After the medium in the culture dish was replaced with Ringer's solution, samples were illuminated either using a mercury lamp for wide-field illumination of using a laser line for TIRF illumination. Setting the correct TIRF angle to isolate the signal from the plasma membrane of proteins that are highly retained at intracellular compartments poses a challenge. To reduce contamination from signals coming from intracellular compartments, the cells were cotransfected with a membrane marker (DsRed-tagged N-terminal region of GAP-43, Clontech) that has an excitation maximum at $557 \mathrm{~nm}$. The critical angle of illumination was set first by monitoring the membrane marker, excited with a $560 \mathrm{~nm}$ laser (Melles-Griot), and then the YFP-KCNQ3 reporter was excited with the $488 \mathrm{~nm}$ line of an argon laser. Changing the excitation to a shorter wavelength reduces the depth of the evanescent wave, improving the isolation of the signal from the plasma membrane. A double valley dichroic mirror (GFP/DsRed, Chroma) was used to allow sequential excitation of both fluorophores without having to move any component of the excitation pathway. Emission filters were 536/40 (Chroma), for YFP and BA590 (Nikon) for DsRed.

Fluorescent images were collected and processed with a 16 bit, cooled charge-coupled camera (model C9100) using the provided Wasabi software (Hamamatsu). This camera uses a front-illuminated EMCCD with on-chip multiplication gain. All camera settings were kept constant for all images. The exposure time was fixed at 750 and $300 \mathrm{~ms}$ for TIRF and epifluorescence, respectively. Analysis was performed using ImageJ software (Version WCIF, National Institutes of Health) after background subtraction. Images were not binned, with pixel size corresponding to a square of $112 \times 112 \mathrm{~nm}$. Cells with similar levels of expression were selected for analysis. Image acquisition and analysis was done blindly, without knowing what construct was transfected. There was no statistical difference averaged pixel intensity of epifluorescence images of the cells included in the comparisons of Figure 2, $B$ and $C$.

\section{Results}

Threonine is the residue most often found at the inner pore vestibule of $\mathrm{K}^{+}$channels, three positions before the GYG signature sequence of the selectivity filter. However, the alignment of the pore region shows that the equivalent residue at position 315 in tetrapod KCNQ3 is an alanine (Fig. $1 A$ ). We substituted threonine for Ala315 in human KCNQ3 and coexpressed this mutant subunit $\left(\mathrm{KCNQ}^{*}\right)$ with KCNQ3. The currents were approximately threefold larger than those produced by coexpression of KCNQ2 with KCNQ3 (Fig. 1C,D).

Since KCNQ3 and KCNQ2 subunits coassemble in equal stoichiometric proportions in neurons (Hadley et al., 2003), we wondered whether $\mathrm{KCNQ}^{*}$ would have any preference to assemble with KCNQ2 over itself. The molecular composition of KCNQ channels can be elucidated by their sensitivity to the potassium channel blocker TEA (Hadley et al., 2003; Etxeberria et al., 2004). The sensitivity of heteromeric KCNQ2/3 is intermediate to that of homomeric KCNQ2 (with high affinity, $\mathrm{EC}_{50} \sim 0.2 \mathrm{mM}$ ) and homomeric KCNQ3 channels (with low affinity, $\mathrm{EC}_{50}>200$ $\mathrm{mm})$. The fit of a Hill equation to the dose-response relation obtained with KCNQ2/3 heteromers was consistent with a single population of channels with an $\mathrm{IC}_{50}=4.1 \mathrm{~mm}$, in accordance with previous reported values (Etxeberria et al., 2004). If the assembly of subunits is a random process, according to the binomial distribution the expected proportion of homomeric channels should be $\sim 6 \%$. Since the maximal current of homomeric $\mathrm{KCNQ}^{*}$ channels is approximately twice of that of KCNQ2/3* 
A

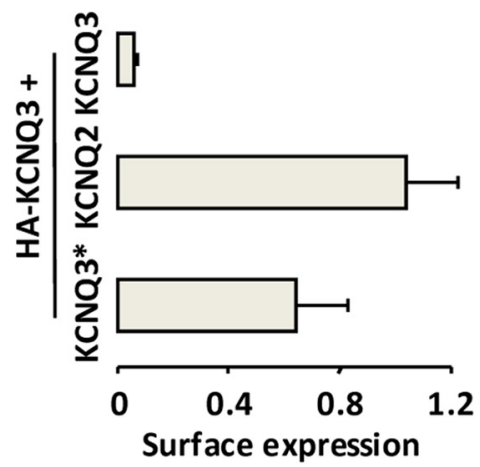

B

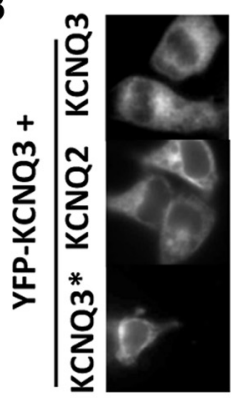

Wide field
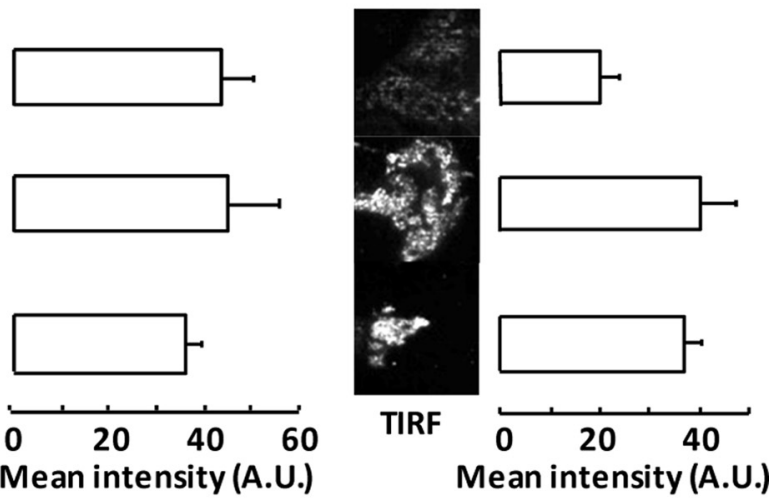

C

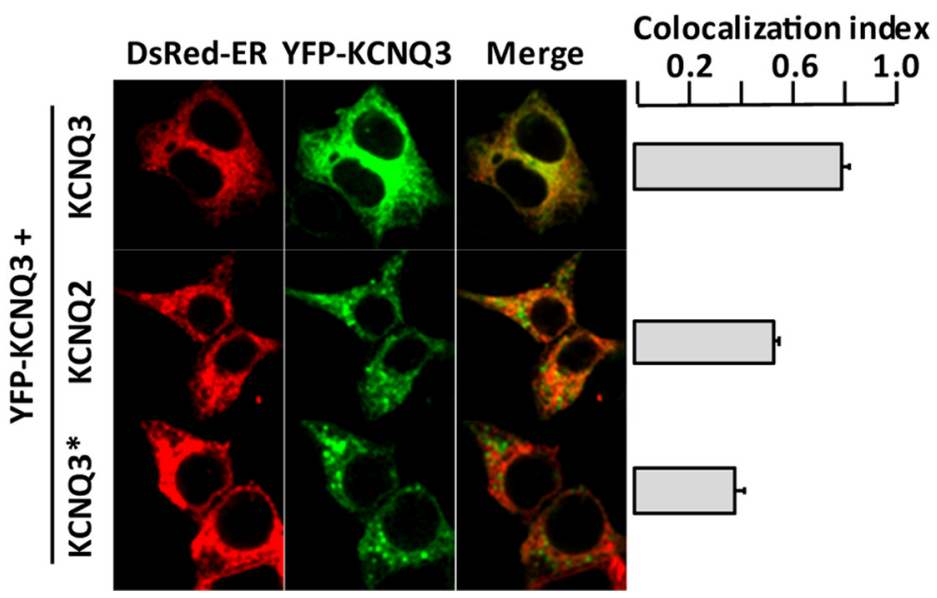

Figure 2. The presence of alanine 315 in KCNQ3 led to ER retention and reduced surface expression. $\boldsymbol{A}$, Normalized surface expression of KCNQ3 subunits tagged with HA at the extracellular S1-S2 loop after coexpression in Xenopus oocytes with the constructs indicated on the left ( $n>15$, three batches of oocytes). The difference in surface expression between KCNQ3/ 2 and KCNQ3/3* heteromers was not statistically significant ( $p>0.6$, unpaired $t$ student test). $\boldsymbol{B}$, Representative TIRF imaging experiments used to quantify membrane expression of KCNQ channels in HEK293T cells. First column, Images under epifluorescence illumination of cells coexpressing YFP-tagged KCNQ3 and the indicated construct. Second column, Averaged epifluorescence intensity. There was no difference in the intensity of wide field epifluorescence images of the cells analyzed. Third column, Fluorescent images under TIRF illumination. Right panel, Quantification of TIRF emission. The emission was quantified by measuring the mean pixel intensity of the outline of the cell for each image, with the background intensity in a cell-free area subtracted. The camera exposure time, laser intensity, and TIRF angle setting remained the same for each cell. Bars show summarized data for each condition, plotted as the mean pixel intensity on the ordinate ( $n \geq 11)$. Statistical analysis of TIRF images, KCNQ3 versus KCNQ2 and KCNQ3 versus KCNQ3* ${ }^{*}, p<0.01$, unpaired $t$ test. C, Effect of coexpression of KCNQ3, KCNQ2, and KCNQ3* on the intracellular distribution of YFP-tagged KCNQ3 subunits. Single-layer confocal images of HEK293T cells cotransfected with the indicated subunits and a DsRed-tagged ER marker. Images with indistinguishable YFP emission intensity levels were analyzed. As reported by others, confocal images did not allow detection of channels at the plasma membrane (Soldovieri et al., 2006). The signals from YFP and DsRed were pseudocolored in green and red, respectively, resulting in a yellow color when they colocalized. The bars on the right represent averaged Manders coefficient of colocalization of cells expressing the different constructs indicated on the left ( $n>24)$. Statistical analysis, KCNQ3 versus KCNQ2 and KCNQ3 versus KCNQ3*,$p<0.00001$; KCNQ2 versus KCNQ3*,$p<$ 0.001 , unpaired $t$ test.
(Etxeberria et al., 2008), the contribution to the current of homomeric $\mathrm{KCNQ} 3^{*}$ channels would increase to $12 \%$. However, the TEA sensitivity suggests that the number of homomeric KCNQ3* was larger. The fit to the dose responses from cells coexpressing $\mathrm{KCNQ}^{*}$ and $\mathrm{KCNQ} 2$ improved by fitting a two-population Hill equation (Fig. $1 E$ ), consistent with $31 \%$ being homomeric $\mathrm{KCNQ}^{*}\left(\mathrm{IC}_{50}=239\right.$ $\mathrm{mM})$, and the remaining, heteromeric $\mathrm{KCNQ} 2 / 3^{\star}$ assemblies $\left(\mathrm{IC}_{50}=6.1 \mathrm{~mm}\right)$. Thus, KCNQ3* does not appear to have a preference to assemble with KCNQ2 over $\mathrm{KCNQ}^{*}$. Given that functional KCNQ3 homotetramers have not been detected in neurons, we conclude that Ala315 plays a crucial role in directing the preferential assembly of KCNQ2/KCNQ3 heteromers.

The unitary current amplitude of $\mathrm{KCNQ}^{*}$ is slightly smaller than that of wild-type KCNQ3 channel, and the open probability is unchanged, while the current density is increased 25-fold (Zaika et al., 2008). The increase in current density could thus be due to activation of previously silent channels already present at the membrane or to an increase in the number expressed at the surface. To investigate this, we used a KCNQ3 subunit tagged with an HA epitope at the extracellular loop between transmembrane 1 and 2 as a reporter of surface expression in Xenopus oocytes. Xenopus oocytes are an excellent model to study KCNQ surface expression, since this expression system faithfully reproduces the increase in KCNQ3 plasma membrane expression observed in neurons after heteromerization with KCNQ2 (Schwake et al., 2000; Etxeberria et al., 2004; Chung et al., 2006). As shown in Figure $2 A$, there was a sixfold increase in the number of KCNQ3 subunits at the plasma membrane when they were coexpressed with $\mathrm{KCNQ}^{*}$. The lack of correlation in surface expression and current levels seen in Figures $1 D$ and $2 A$ are likely to be due to the reduced conductance of KCNQ2/3* heteromers (Etxeberria et al., 2008) and to the contribution to the current of homomeric KCNQ3 ${ }^{*}$ channels (that are not detected in the surface expression assay).

Our findings are in contrast with those of a recent report that did not find a significant increase in surface expression when this mutant subunit was expressed in a mammalian cell line (Zaika et al., 2008). We, therefore, studied trafficking in human HEK293T cells by TIRF microscopy. TIRF microscopy enabled us to visualize the impact of coexpression of other subunits on YFP-KCNQ3 plasma 
membrane targeting. With TIRF microscopy, it is possible to excite only fluorophores located within $\sim 300 \mathrm{~nm}$ or less of the plasma membrane. Any molecule deeper in the cytoplasm will not be illuminated. As mentioned above, it is well established that surface expression of KCNQ3 subunits increases after heteromerization with KCNQ2 in neurons and oocytes (Schwake et al., 2000; Etxeberria et al., 2004; Chung et al., 2006). However, a recent report has not found significant differences in the mean TIRF intensity of images from $\mathrm{CHO}$ cells expressing KCNQ3 or KCNQ3+KCNQ2 (Zaika et al., 2008). Why Zaika et al. (2008) did not see any increase in surface expression is unclear to us. It could be for technical reasons or due to unexplained differences in the behavior of the cells. Quantification of surface expression of KCNQ channels is especially challenging, since most of the subunits are located intracellularly. A minimal contribution from the intracellular signal will lead to an underestimation of surface expression. We used a longer wavelength of excitation to establish the angle of illumination to achieve TIRF conditions, and then switched to a shorter wavelength for image acquisition, reducing the depth of the evanescent wave, therefore reducing the background from intracellular signals. In our experiments, the TIRF signal clearly increased when YFP-KCNQ3 subunits were coexpressed with KCNQ2 (Fig. 2B), a result consistent with the surface expression results (Fig. $2 A$ ). The TIRF signal was also greater when the mutant $\mathrm{KCNQ}^{*}$ subunit was coexpressed with wild-type KCNQ3, indicating that the presence of the $\mathrm{A} 315 \mathrm{~T}$ mutation at the inner vestibule of KCNQ3-containing heterotetramers enhances channel trafficking to the plasma membrane in both amphibian and mammalian cells.

To identify the intracellular location of YFP-KCNQ3, it was coexpressed with a red emitting fluorescent ER marker (Etxeberria et al., 2008; Alaimo et al., 2009). After coexpression with KCNQ2 or KCNQ3 ${ }^{*}$, conspicuous puncta of intense YFPKCNQ3 signal appeared, whereas those puncta were absent in the cells coexpressing KCNQ3. The apparition of those densities correlated with the increased surface expression seen in oocytes, suggesting that they are related to channels trafficking to or from the plasma membrane. The colocalization analysis from cells with similar YFP-KCNQ3 expression levels revealed that most of the YFP-KCNQ3 subunits were retained within this compartment when they were coexpressed with KCNQ3. Retention was reduced, however, by coexpression of either KCNQ2 or KCNQ3* subunits (Fig. 2C). These results demonstrate that assembly with KCNQ2 or KCNQ3* promotes ER exit of KCNQ3 subunits.

It has been shown that the residues located in the inner vestibule of AMPA receptors have an impact on ER exit by influencing the formation of tetramers (Greger et al., 2003). By analogy, the residue at position 315 may influence assembly of KCNQ3 channels. This was analyzed first by performing coimmunoprecipitation experi-
B

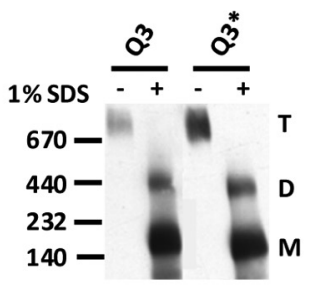

C

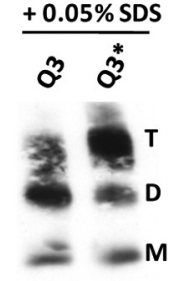

Figure 3. KCNQ3 and KCNQ3* do not differ in their capacity to form tetramers. $\boldsymbol{A}$, Coimmunoprecipitation of HA-tagged KCNQ3 and KCNQ3* assembled with YFP-tagged KCNQ3 subunits. As a negative control, a sample expressing untagged KCNQ3 coexwa-KCNQ3 was processed simultaneously (column labeled as (trl). Proteins from HEK293T cells expressing the

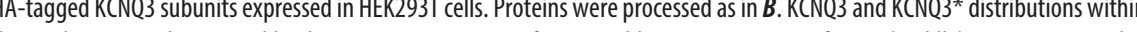
mple from fraction 16 was treated with $1 \%$ SDS for 60 min at $37^{\circ} \mathrm{C}$ to dissociate the larger complexes; the resulting product was designated as monomer (right). HA signals from fractions 9-16 were summed up and set at $100 \%$ (total):individual signalsineach fraction were expressed as percentage of the total $(n=2)$.

ments, which did not reveal any major impact on the ability of KCNQ3 to dimerize with KCNQ3 or KCNQ3 ${ }^{*}$ (Fig. 3A). We next analyzed channel complexes by BN-PAGE. KCNQ3 and KCNQ3* tetramers were detected at the expected molecular size (Fig. $3 B$ ). Bands corresponding to tetramers (and dimers) were resolved in all the coexpression experiments with KCNQ2, KCNQ3, or KCNQ3* (supplemental Fig. S2, available at www.jneurosci.org as supplemental material). These data suggest that KCNQ3 can efficiently assemble into tetramers with itself and with KCNQ2 or KCNQ3* .

We noticed the presence of bands corresponding to monomers that were more intense for extracts from cells expressing KCNQ3 homotetramers (supplemental Fig. S2, available at www. jneurosci.org as supplemental material), suggesting that these complexes were less resistant to SDS treatment. Further experiments were performed using different concentrations of SDS. KCNQ3 tetramers were resolved after incubation with $0.025 \%$ SDS (supplemental Fig. S3, available at www.jneurosci.org as supplemental material). Following incubation with $0.05 \%$ SDS, $\mathrm{KCNQ}^{*}$ tetramers were fairly resistant to dissociation, although dimers and monomers were also resolved. In contrast, the majority of KCNQ3 was resolved as a dimer (Fig. 3C). These results suggest that, although the wild-type subunits can assemble as homomultimers, the stability of the homotetramers is improved by the A315T substitution.

In addition to the susceptibility to SDS, there was a remarkable difference between KCNQ3 and $\mathrm{KCNQ}^{*}$ complexes. Al- 
A
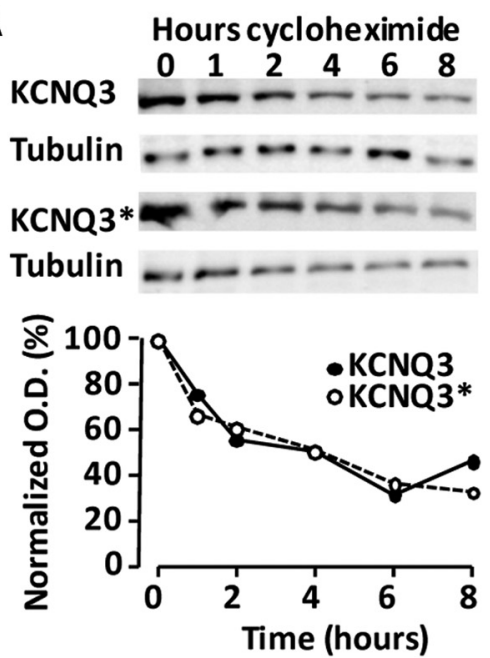

B

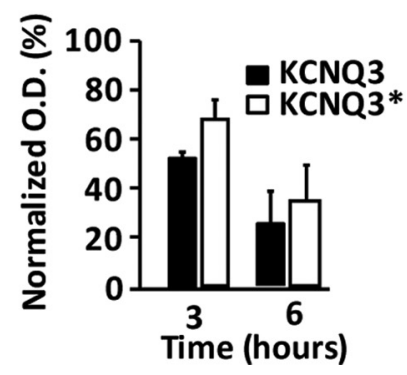

C

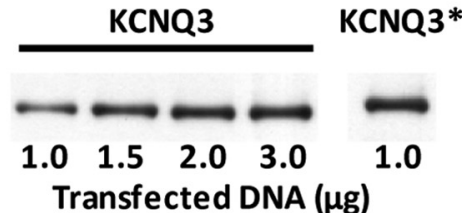

D

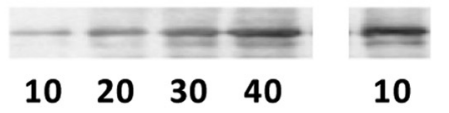

Sample volume $(\mu \mathrm{L})$

E

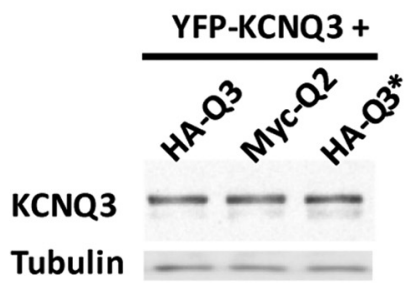

The expectation is that only correctly assembled homotetramers will traffic to the plasma membrane via the Golgi apparatus, whereas aggregated complexes will be retained in the ER and subsequently degraded. However, the larger complexes observed might not be due to gross misfolding of the protein. If this is the case, this effect will not be reflected in changes in the susceptibility to degradation. Indeed, treatment of cells with the protein synthesis inhibitor cycloheximide did not reveal a significant difference in the rate of degradation (Fig. 4A,B). Quantitative analysis of protein expression revealed that the yield of $\mathrm{KCNQ}^{*}$ was consistently approximately four times larger than that of wt KCNQ3 (Fig. 4C,D). We addressed the impact of assembly on protein yield by coexpressing KCNQ3 subunits with different partners (KCNQ3, KCNQ2, and $\mathrm{KCNQ}^{*}$ ) at $1: 1,1: 2$, and $1: 3$ ratios to gradually increase the chances of incorporating partner subunits into heteromers. These experiments did not reveal any significant difference on KCNQ3 protein levels (Fig. 4E). Thus, in contrast to the impact on ER exit, heteromerization did not influence this process, indicating that the mechanisms by which Ala315 affect protein yield and ER exit are different.

Figure 4. The A315T mutant presents increased protein yield. Tubulin was monitored to adjust sample load. Channel subunits were detected using an anti-HA monoclonal antibody $(\boldsymbol{A}-\boldsymbol{D})$ or anti-GFP $(\boldsymbol{E})$. $\boldsymbol{A}$, Time course analysis (hours after addition of $50 \mu \mathrm{M}$ cycloheximide) of KCNQ3 and KCNQ3* protein levels by Western blot of total cell lysates from HEK293T cells. Bottom, Data expressed as percentage of optical density, obtained after densitometric analysis of the bands corresponding to wild-type and mutant KCNQ3. $\boldsymbol{B}$, Relative optical density to the value at time $=0$ of extracts from cells expressing the indicated subunits after 3 or $6 \mathrm{~h}$ of treatment with $50 \mu \mathrm{m}$ cycloheximide $(n=4)$. The differences on relative optical density between KCNQ3 and KCNQ3* taken at the same time points were not statistically significant. $C$, Western blot using anti-HA of total cell lysates from HEK293T transfected with increasing amount of DNA encoding KCNQ3 compared to extracts from cells transfected with $1 \mu \mathrm{g}$ of DNA encoding KCNQ3* (right) $(n=3)$. $\boldsymbol{D}$, Western blot using anti-HA of increasing sample load from cells expressing the same amount of DNA ( $1 \mu \mathrm{g}$ ) coding KCNQ3 subunits compared to KCNQ3* in HEK293T cells. The optical density (OD) of the bands was analyzed using ImageJ. A linear regression fit was obtained from the $0 D$ v s load relation. From this regression, the amount required to obtain the same ODs was found to be $4.07 \pm 0.28$-fold larger $(n=3)$ for KCNQ3 subunits. $\boldsymbol{E}$, Western blot probed with anti-GFP of cells cotransfected with YFP-KCNQ3 and the indicated partner at a 1:3 ratio. No differences in the intensity of the band corresponding to YFP-KCNQ3 were found. Similar results were obtained when the ratio was 1:1 or 1:2 $(n=3)$.

though the same amount of protein was loaded in the BN gel, the band corresponding to KCNQ3 tetramers was less intense than that of KCNQ3* tetramers (Fig. 3B). A smear at higher molecular weight was observed (see supplemental Fig. S3, available at www. jneurosci.org as supplemental material). This may reflect tetramers bound to other proteins, such as chaperones, folding intermediates, or aggregates of tetramers that were retained at the stacking gel. Supporting the idea that those smears contained tetramers, we found an increase in the intensity of the tetramer band after incubation with $0.025 \%$ SDS (see supplemental Fig. S3, available at www.jneurosci.org as supplemental material). To further confirm the oligomeric state of KCNQ3 and KCNQ3* homomers, their hydrodynamic behavior was analyzed. Cell extracts were fractionated on a continuous (10-50\%) glycerol gradient and gradient fractions were analyzed by BN Western blot. Compared to $\mathrm{KCNQ}^{*}$, KCNQ3 channels were accumulated in the most dense fraction (fraction 16), which corresponds to larger complexes, and were less abundant in the lighter fraction (fraction 9), that corresponds to tetramers (Fig. 3D).

\section{Discussion}

Among eukaryotic potassium channels, the KCNQ3 subunit is unusual because it has an alanine at the pore inner vestibule, three residues before the GYG signature sequence of the selectivity filter (Fig. 1A). We previously identified Ala315 in KCNQ3 as a critical signal for M-current potentiation (Etxeberria et al., 2004). Here, we have shown a clear impact of Ala315 on KCNQ3 channel trafficking. The effect of this residue is to ensure the preferential expression of KCNQ2/3 heteromers rather than KCNQ3 homotetramers at the plasma membrane, playing a crucial role in determining the nature of the native neuronal channels.

Several studies have shown that the number of channels at the cell surface increases after heteromerization of KCNQ2 and KCNQ3 subunits in oocytes and neurons (Schwake et al., 2000; Etxeberria et al., 2004; Chung et al., 2006). In contrast, a recent report did not detect such increase and the TIRF data did not reveal differences in surface expression for the A315T mutant either (Zaika et al., 2008). In our experiments, the TIRF signal clearly increased when KCNQ3 subunits were coexpressed with KCNQ2 or with the A315T mutant, a result consistent with the surface expression results, ER colocalization data, and with previous reports.

It was generally thought that the pore does not exert a major role in M-channel surface expression (Maljevic et al., 2003; Schwake et al., 2003, 2006; Etxeberria et al., 2004; Nakajo and Kubo, 2008; Zaika et al., 2008). Therefore, the finding that this 
residue is a major KCNQ3 trafficking determinant is clearly surprising. However, the concept of surface expression regulation by the pore is not new, since the reentrant $\mathrm{P}$ loops on AMPA receptor have been identified as traffic determinants (Greger et al., 2003).

How can a residue buried in the core of the protein control traffic? How is it recognized by the transport machinery? Structural data are required to understand our findings fully. The only other human potassium channel that we have found with an alanine at this position is Kir3.1, encoded by gene KCNJ3. The atomic structure of a chimeric channel with the pore region from eukaryotic Kir3.1 (Fig. 1B) showed that, compared to KcsA (which has a threonine), the three-dimensional structure of the selectivity filter is conserved within $0.1-0.2 \AA$ (Nishida et al., 2007). However, the protein core surrounding the selectivity filter forms a highly interdependent network of amino acids, and differences on the sequence at other positions may have an impact on the structure. Molecular modeling indicates that the critical factor for the position 315 is not hydrophobicity per se, but rather the stabilization of the selectivity filter by virtue of the creation of a mininetwork of hydrogen bonds. Without this stabilization, the selectivity filter would be unstable and likely to collapse (Zaika et al., 2008). In line with this model, we have found that KCNQ3 multimeric channels are disrupted after mild SDS treatment into dimers and monomers more readily than the A315T mutant channels. In addition, our data suggest that the inherent instability of KCNQ3 homomeric channels may be responsible for their retention in the ER and subsequent low cell surface delivery. As it has been demonstrated in yeast (Kowalski et al., 1998), it is likely that the instability leads to the observed ER retention. The creation of a more favorable hydrogen bond network is also the most probable mechanism to explain the increased yield of the A315T mutant, since mutations in GFP that result in more favorable hydrogen bonding have been found to increase stability, folding rate, and yield (Sanders and Jackson, 2009). However, in contrast to the lack of effect on protein yield, assembly with KCNQ3-A315T resulted in ER exit and in a sixfold increase in surface delivery of KCNQ3.

Does the impact on trafficking accounts for the reported 25fold increase in current density for the KCNQ3-A315T mutant (Zaika et al., 2008)? This extent of increase would imply that at least $96 \%$ of KCNQ3 channels are retained intracellularly. If we take into account the fourfold increase in protein yield seen in the A315T mutant, that figure will become less stringent (at least 75\% of the channels should be intracellular). The surface expression results obtained in oocytes and reported for neurons (Chung et al., 2006) are compatible with these figures and the confocal images of HEK293T cells expressing fluorescently tagged subunits show a predominant intracellular distribution.

The ability of the mutant KCNQ3-A315T subunit to promote exit of wild-type KCNQ3 subunits from the ER indicates that threonine at position 315 in only some of the subunits within the complex is sufficient to overcome the retention mechanism. This is not, however, the only means of promoting KCNQ3 exit. It is well established that heteromerization of the $\mathrm{C}$ terminus plays a role on the surface expression increase after KCNQ2 and KCNQ3 assembly (Maljevic et al., 2003; Schwake et al., 2006; Nakajo and Kubo, 2008). Previously we showed that a chimeric subunit in which the $\mathrm{C}$ terminus of KCNQ3 was substituted for that of KCNQ2 also promoted the efficient traffic of KCNQ3 (Etxeberria et al., 2004), suggesting that a complex in which all subunits have alanine within the pore inner vestibule can still escape retention. However, those chimeric channels were nonfunctional, suggest-

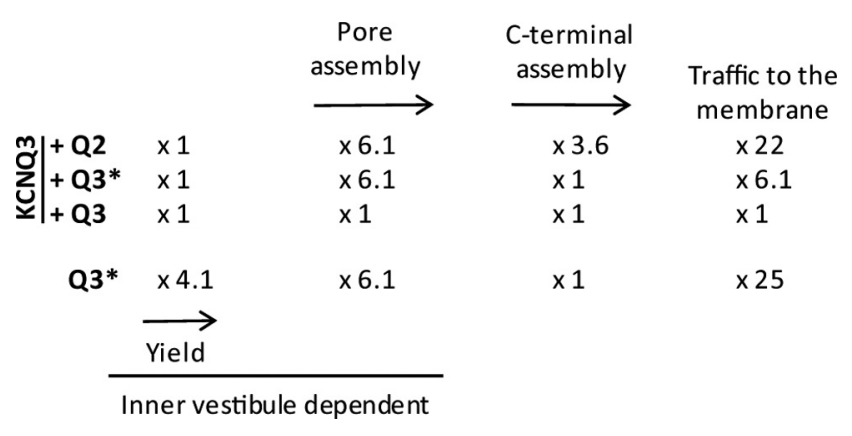

Figure 5. Model for trafficking control. Alanine 315 controls trafficking by two separable steps. The first involves protein yield, and the second step is related to the stability of assembled subunits. In KCNQ3* the rate of both steps are increased by a factor of 4.1 and 6.1, respectively, resulting in an overall 25 -fold increase in channel density at the plasma membrane. Heteromerization with KCNQ2 does not affect the first step, but results in tighter assembly, and engages another mechanism that involves the C-terminal region. The rates of the second and third steps are increased by factors of 6.1 and 3.6, respectively, resulting in a 22-fold increase in density upon heteromerization. The final increase in channel density has been taken from (Zaika et al., 2008). The impact on ER retention was then calculated for KCNQ3*, taking into account the fourfold increase in protein yield. The impact of the C terminus was calculated assuming the previous figure for ER retention. The resulting figure (3.6) is similar to the reported impact on KCNQ3 surface expression of a cytosolic fragment of helix D from KCNQ2 (Schwake et al., 2006).

ing that the configuration of the pore with four alanines was blocked or collapsed (Etxeberria et al., 2004; Zaika et al., 2008). The involvement of the $\mathrm{C}$ terminus of KCNQ2 would explain why surface expression was larger when KCNQ3 subunits were coexpressed with KCNQ2 than when coexpressed with KCNQ3A315T (see Fig. 2).

As for other potassium channels, assembly of M-channels is thought to be a two step process initiated by subunit dimerization, followed by tetramerization via dimerization of dimers (Papazian, 1999). Our results are in line with this model, as dimers and tetramers are isolated readily; trimers however, are not detected. Although KCNQ3 has a strong tendency to form high-molecular-weight complexes, our finding that this residue has a negligible impact on the degradation rate argues against gross misfolding, as misfolded proteins are rapidly degraded by ER-associated degradation mechanisms (ERAD) (Vembar and Brodsky, 2008).

It has to be kept in mind that, like other ion channels, KCNQ is a modular protein, and that the pore region oligomeric state may differ from that of the intracellular C-terminal assembly domain at a given instant. It should also be taken into consideration that many tetrameric $\mathrm{K}^{+}$channels are devoid of so called "assembly domain." On the other hand, the isolated C-terminal "assembly domain" of KCNQ forms tetramers spontaneously in vitro (Howard et al., 2007; Wehling et al., 2007; Wiener et al., 2008). It has been suggested that monomers, dimers, and tetramers of AMPAR are in a dynamic equilibrium, and that the pore determines the balance between these intermediates (Greger et al., 2003). We envisage the following scenario for the ER-exit of KCNQ channels. It seems plausible that oligomerization of the transmembrane domain and the C-terminal region are mostly independent processes. We suggest that the channel can exit the ER only when both the pore region and the C-terminal assembly domain are forming tetramers. In addition, a channel with only one of these two domains tetramerized and with the other domain unassembled may not be targeted for degradation, explaining the similar degradation rates of wt and the A315T mutant. Assembly with KCNQ3-A315T shifts the equilibrium for the pore domain between dimers and tetramers to the right, resulting in 
ER exit and in a sixfold increase in surface delivery. On the other hand, assembly of the C-terminal region with helix D of KCNQ2 results in an additional 3.6-fold increase in plasma membrane expression (Schwake et al., 2006). Thus, when KCNQ3 assembles with KCNQ2 the final increase in surface would be 22 -fold, i.e., the product of the contribution of the pore and assembly domains (see Fig. 5).

We have found an alanine at the position equivalent to residue 315 of human KCNQ3 in every tetrapod ortholog, but not in the four ortholog sequences from fishes available to date (see supplemental Fig. S1, available at www.jneurosci.org as supplemental material). Therefore, KCNQ3 A315 may represent a tetrapodspecific divergence, as almost all Kv and all earlier KCNQ subunits posses a $\mathrm{T}$ at the equivalent position. There is general agreement on that the ancestor of tetrapods was a lobe-finned fish (Joss, 2006). However, it is not known how close either of the extant groups of lobe-finned fish, lungfish or coelacanths, is to the actual ancestor. We believe that the study of the KCNQ3 pore sequence in coelacanths and lungfishes will shed new light on the evolutionary transition from fish to tetrapod.

In summary, the presence of alanine at the inner vestibule makes the function of tetrapod KCNQ3 subunits dependent on the assembly with KCNQ2 subunits, and, in conjunction with a relatively secondary role of the $\mathrm{C}$ terminus, favors the traffic of channels with a 1:1 stoichiometry to the plasma membrane. Heteromers may be advantageous as they allow more diverse regulation through action upon distinct KCNQ2 and KCNQ3 components.

Note added in proof. A recent report presents crystallographic evidence for structural connections between the inner vestibule and cytosolic domains of KirBac3.1 potassium channels (Clarke et al., 2010). The transport machinery might recognize structural rearrangements in cytosolic domains of KCNQ3 channels transmitted from the inner vestibule by mechanisms analogous to those described for KirBac3.1.

\section{References}

Alaimo A, Gómez-Posada JC, Aivar P, Etxeberría A, Rodriguez-Alfaro JA, Areso P, Villarroel A (2009) Calmodulin activation limits the rate of KCNQ2 $\mathrm{K}^{+}$channel exit from the endoplasmic reticulum. J Biol Chem 284:20668-20675.

Chung HJ, Jan YN, Jan LY (2006) Polarized axonal surface expression of neuronal KCNQ channels is mediated by multiple signals in the KCNQ2 and KCNQ3 C-terminal domains. Proc Natl Acad Sci U S A 103:8870-8875.

Clarke OB, Caputo AT, Hill AP, Vandenberg JI, Smith BJ, Gulbis JM (2010) Domain reorientation and rotation of an intracellular assembly regulate conduction in Kir potassium channels. Cell 141:1018-1029.

Costes SV, Daelemans D, Cho EH, Dobbin Z, Pavlakis G, Lockett S (2004) Automatic and quantitative measurement of protein-protein colocalization in live cells. Biophys J 86:3993-4003.

Etxeberria A, Santana-Castro I, Regalado MP, Aivar P, Villarroel A (2004) Three mechanisms underlie KCNQ2/3 heteromeric potassium M-channel potentiation. J Neurosci 24:9146-9152.

Etxeberria A, Aivar P, Rodriguez-Alfaro JA, Alaimo A, Villacé P, GómezPosada JC, Areso P, Villarroel A (2008) Calmodulin regulates the trafficking of KCNQ2 potassium channels. FASEB J 22:1135-1143.

Greger IH, Khatri L, Kong X, Ziff EB (2003) AMPA receptor tetramerization is mediated by $\mathrm{Q} / \mathrm{R}$ editing. Neuron 40:763-774.

Hadley JK, Passmore GM, Tatulian L, Al-Qatari M, Ye F, Wickenden AD, Brown DA (2003) Stoichiometry of expressed KCNQ2/KCNQ3 channels and subunit composition of native ganglionic M-currents deduced from block by tetraethylammonium (TEA). J Neurosci 23:5012-5019.

Hill AS, Nishino A, Nakajo K, Zhang G, Fineman JR, Selzer ME, Okamura Y, Cooper EC (2008) Ion channel clustering at the axon initial segment and node of Ranvier evolved sequentially in early chordates. PLoS Genet 4:e1000317.
Howard RJ, Clark KA, Holton JM, Minor DL Jr (2007) Structural insight into KCNQ (Kv7) channel assembly and channelopathy. Neuron 53:663-675.

Jentsch TJ (2000) Neuronal KCNQ potassium channels: physiology and role in disease. Nat Rev Neurosci 1:21-30.

Joss JM (2006) Lungfish evolution and development. Gen Comp Endocrinol 148:285-289.

Kowalski JM, Parekh RN, Mao J, Wittrup KD (1998) Protein folding stability can determine the efficiency of escape from endoplasmic reticulum quality control. J Biol Chem 273:19453-19458.

Maljevic S, Lerche C, Seebohm G, Alekov AK, Busch AE, Lerche H (2003) C-terminal interaction of KCNQ2 and KCNQ3 $\mathrm{K}^{+}$channels. J Physiol 548:353-360.

Maljevic S, Wuttke TV, Lerche $\mathrm{H}$ (2008) Nervous system $\mathrm{K}_{\mathrm{V}} 7$ disorders: break down of a subthreshold brake. J Physiol 586:1791-1801.

Manders EMM, Stap J, Brakenhoff GJ, van Driel R, Aten JA (1992) Dynamics of 3-dimensional replication patterns during the S-phase, analyzed by double labeling of DNA and confocal microscopy. J Cell Sci 103:857-862.

Misonou H, Trimmer JS (2004) Determinants of voltage-gated potassium channel surface expression and localization in mammalian neurons. Crit Rev Biochem Mol Biol 39:125-145.

Nakajo K, Kubo Y (2008) Second coiled-coil domain of KCNQ channel controls current expression and subfamily specific heteromultimerization by salt bridge networks. J Physiol 586:2827-2840.

Nishida M, Cadene M, Chait BT, MacKinnon R (2007) Crystal structure of a Kir3.1-prokaryotic Kir channel chimera. EMBO J 26:4005-4015.

Pan Z, Kao T, Horvath Z, Lemos J, Sul JY, Cranstoun SD, Bennett V, Scherer SS, Cooper EC (2006) A common ankyrin-G-based mechanism retains $\mathrm{KCNQ}$ and $\mathrm{NaV}$ channels at selectively active domains of the axon. J Neurosci 26:2599-2613.

Papazian DM (1999) Potassium channels: some assembly required. Neuron 23:7-10.

Rasmussen HB, Frøkjaer-Jensen C, Jensen CS, Jensen HS, Jørgensen NK, Misonou H, Trimmer JS, Olesen SP, Schmitt N (2007) Requirement of subunit co-assembly and ankyrin-G for M-channel localization at the axon initial segment. J Cell Sci 120:953-963.

Sanders JK, Jackson SE (2009) The discovery and development of the green fluorescent protein, GFP. Chem Soc Rev 38:2821-2822.

Schroeder BC, Kubisch C, Stein V, Jentsch TJ (1998) Moderate loss of function of cyclic-AMP-modulated KCNQ2/KCNQ3 $\mathrm{K}^{+}$channels causes epilepsy. Nature 396:687-690.

Schwake M, Pusch M, Kharkovets T, Jentsch TJ (2000) Surface expression and single channel properties of KCNQ2/KCNQ3, M-type $\mathrm{K}^{+}$channels involved in epilepsy. J Biol Chem 275:13343-13348.

Schwake M, Jentsch TJ, Friedrich T (2003) A carboxy-terminal domain determines the subunit specificity of $\mathrm{KCNQ} \mathrm{K}^{+}$channel assembly. EMBO Rep 4:76-81.

Schwake M, Athanasiadu D, Beimgraben C, Blanz J, Beck C, Jentsch TJ, Saftig P, Friedrich T (2006) Structural determinants of M-type KCNQ (Kv7) $\mathrm{K}^{+}$channel assembly. J Neurosci 26:3757-3766.

Shah MM, Mistry M, Marsh SJ, Brown DA, Delmas P (2002) Molecular correlates of the M-current in cultured rat hippocampal neurons. J Physiol 544:29-37.

Soldovieri MV, Castaldo P, Iodice L, Miceli F, Barrese V, Bellini G, Miraglia del Giudice E, Pascotto A, Bonatti S, Annunziato L, Taglialatela M (2006) Decreased subunit stability as a novel mechanism for potassium current impairment by a KCNQ2 C terminus mutation causing benign familial neonatal convulsions. J Biol Chem 281:418-428.

Vembar SS, Brodsky JL (2008) One step at a time: endoplasmic reticulumassociated degradation. Nat Rev Mol Cell Biol 9:944-957.

Wang HS, Pan Z, Shi W, Brown BS, Wymore RS, Cohen IS, Dixon JE, McKinnon D (1998) KCNQ2 and KCNQ3 potassium channel subunits: molecular correlates of the M-channel. Science 282:1890-1893.

Wehling C, Beimgraben C, Gelhaus C, Friedrich T, Saftig P, Grötzinger J, Schwake M (2007) Self-assembly of the isolated KCNQ2 subunit interaction domain. FEBS Lett 581:1594-1598.

Wiener R, Haitin Y, Shamgar L, Fernández-Alonso MC, Martos A, ChomskyHecht O, Rivas G, Attali B, Hirsch JA (2008) The KCNQ1 (Kv7.1) $\mathrm{COOH}$ terminus, a multitiered scaffold for subunit assembly and protein interaction. J Biol Chem 283:5815-5830.

Zaika O, Hernandez CC, Bal M, Tolstykh GP, Shapiro MS (2008) Determinants within the turret and pore-loop domains of KCNQ3 $\mathrm{K}^{+}$channels governing functional activity. Biophys J 95:5121-5137. 